\title{
Circadian Gene Variations Are Associated with Obsessive-Compulsive Behaviors in a Young Healthy Korean Population
}

\author{
Jaegwon Jeong ${ }^{1,2}$, Yujin Lee ${ }^{1,2}$, Chul-Hyun Cho ${ }^{1,2}$, and Heon-Jeong Lee ${ }^{1,2,3}$ \\ ${ }^{1}$ Department of Psychiatry, Korea University College of Medicine, Seoul, Korea \\ ${ }^{2}$ Chronobiology Institute, Korea University, Seoul, Korea \\ ${ }^{3}$ Department of Biomedical Science, Korea University College of Medicine, Seoul, Korea
}

\begin{abstract}
Objective: The aim of this study is to evaluate the association between obsessive-compulsive behaviors and variations of circadian genes. Methods: We evaluated whether 6 polymorphisms in 5 circadian genes CLOCK, ARNTL, NPAS2, PER2, and PER3 were associated with obsessive behaviors in a Korean population. In all, 292 healthy subjects were genotyped for 6 circadian gene variants. Obsessive-compulsive behaviors were assessed with the Korean version of Maudsley Obsessive-Compulsive Inventory (MOCI). Results: ARNTL rs2278749 polymorphism was significantly associated with obsessive-compulsive behaviors in dominant model. PER3 rs57875989 polymorphism and obsessivecompulsive behaviors showed significant association in recessive and genotypic model. Checking and cleaning subscales of MOCI showed statistically significant difference in ARNTL genotypes. Conclusion: The findings of this study support that circadian rhythm may influence obsessive-compulsive behaviors. Further studies are needed to explain the link between the circadian rhythm and obsessive-compulsive behavior.

Key Words: Circadian rhythms; Polymorphism, genetic; Obsessive behavior; BMAL1 protein, human; PER3 protein, human

Received: September 15, 2019 Revised: September 18, 2019 Accepted: September 18, 2019

Corresponding author: Heon-Jeong Lee, MD, PhD, Department of Psychiatry, Korea University College of Medicine, 73 goryeodae-ro, Seongbuk-gu, Seoul 02841 , Korea.

Tel: 82-2-920-5815, Fax: 82-2-929-7679, E-mail: leehjeong@korea.ac.kr

(a) This is an Open Access article distributed under the terms of the Creative Commons Attribution Non-Commercial License (https://creativecommons.org/licenses/bync/4.0) which permits unrestricted non-commercial use, distribution, and reproduction in any medium, provided the original work is properly cited.
\end{abstract}

\section{INTRODUCTION}

Recently, growing evidence implies that circadian rhythm may influence and/or play a central role in psychiatric disorders, such as bipolar disorder [1,2], schizophrenia [3], and autism [4]. However, there is limited knowledge on the role of circadian rhythms in obsessive-compulsive disorder (OCD).

Disruption of circadian regulation of body temperature and hormonal levels has been observed in those with OCD [5-7]. Difference in sleep behavior (i.e., reduced total sleep time, increased awakening after sleep onset, extended sleep onset latency) and in sleep architecture [i.e., decreased latency to the onset of rapid eye movement (REM) sleep and increased density of REM sleep] have been reported in OCD patients compared to healthy individuals [8-12]. Delayed bedtimes are also associated with increased OC symptoms [13]. Delayed sleep phase disorder is known to be more prevalent in severe OCD patients $[14,15]$. Seasonal affective disorder is also more prevalent in OCD patients, and obsessive symptoms appear to have seasonal fluctuation [16]. Furthermore, eveningness, defined as the tendency to be most active and alert during the evening, also appears to contribute to the development of OCD symptoms [17]. Higher latitude and fewer hours of light exposure have been associated with increased OCD prevalence [18]. These findings suggest that an underlying circadian disturbance may contribute to OCD symptoms and OCD itself.

The molecular mechanisms of circadian rhythm consists of networks of transcriptional-translational feedback loops that give a rhythmic expression pattern to circadian genes (e.g., CLOCK, BMAL1, PER1, PER2, PER3, CRY1, CRY2) [19]. To date, circadian genetic variation studies on human characteristics have been mainly studied in relation to diurnal preferences [20-23] and seasonal variations [24]. A recent study implied that eveningness predicts OCD symptoms over the course of 4 months, controlling for depression [17]. On the other hand, in adolescents, eveningness 
does not predict OCD symptoms over the course of 6 months [25].

As far as we know, there are no previous studies that analyzed the relationship between obsessive behaviors and circadian genes in a normal population. In the present study, we examined the influence of the CLOCK, ARNTL, NPAS2, PER2, and PER3 genes on obsessive behaviors in a young healthy Korean population. We selected CLOCK, ARNTL, NPAS2, PER2, and PER3 due to their importance in circadian rhythm regulations.

\section{METHODS}

\section{Subjects}

Community-based healthy young adults were recruited through social media. Of the 292 participants recruited, 187 were male and 105 were female. The age range of the subjects was $18-35$ years. Participants were confirmed to be free of lifetime or current diagnoses of psychiatric disorders by an experienced psychiatrist using the Mini-International Neuropsychiatric Interview. Subjects with family histories of substance abuse or major psychiatric disorders (e.g., schizophrenia or mood disorders) were excluded. The subjects were unrelated Koreans who were free of major medical problems and lived in Seoul, Korea. All participants gave informed consent, and study protocols were approved by the Institutional Review Board of Korea University Hospital (2014AN0241).

\section{Genotyping}

Genomic DNA was extracted from leukocytes with a QIAamp Blood Kit (Qiagen, Hilden, Germany). Genotyping for the PER3 rs57875989 variable number tandem repeat (VNTR) was performed using polymerase chain reaction (PCR) (forward primer 5'-TGT CTT TTC ATG TGC CCT TAC TT-3' and reverse primer 5'-TGT CTG GCA TTG GAG TTT GA-3') with the following amplification conditions: $94^{\circ} \mathrm{C} / 3 \mathrm{~min}$, then 38 cycles of $94^{\circ} \mathrm{C} / 45$ sec, $58^{\circ} \mathrm{C} / 45 \mathrm{sec}$, and $72^{\circ} \mathrm{C} / 1 \mathrm{~min}$. Agarose gel electrophoresis was used to determine whether individuals were heterozygotic or homozygotic for the PER3 rs57875989 VNTR allele.

Genotyping for five other circadian gene SNPs was performed by high-resolution melting curve analysis. PCR was performed in a volume of $20 \mu \mathrm{L}$ per reaction with a 96-well Bio-Rad CFX 96 Real-Time PCR system (Bio-Rad, Hercules, CA, USA). Reaction mixtures included $1.5 \mu \mathrm{L}$ of genomic DNA as template, $200 \mathrm{mM}$ of each primer [NPAS2 (rs2305160), forward 5'-GAT TTG TGG GAA CTT CTT GAG-3' and reverse 5'-ATG AGG ACT GTT TGA TGT GT-3'; ARNTL (rs2278749), forward 5'-CAT CAT TAC AGC ACC TCC TC-3' and reverse 5'-CCA CTT TTC CTT CCC CAT AG-3'; CLOCK (rs1801260), forward 5'-GGG AAA GTT CCA GCA GTT-3' and reverse 5'-ATC CAG GCA CCT AAA ACA-3'; PER2 (rs2304672), forward 5'-TGT TTG CCA GCT TCG TCC-3' and reverse 5'-GTT GGT CTC ATG TCC ACT-3'; PER2 (rs934945), forward 5'-ATC TCA AGT TTG TTA CTG ATT TCT C-3' and reverse 5'-CTC TTC GAT CCT GTG ATT CAA-3'], 1X SsoFast ${ }^{\mathrm{TM}}$ EvaGreen ${ }^{\circledR}$ Supermix (Bio-Rad Laboratories Inc., Hercules, CA, USA), and sterile $\mathrm{H}_{2} \mathrm{O}$. The amplifi- cation protocol started with $98^{\circ} \mathrm{C} / 3 \mathrm{~min}$ followed by 39 cycles of $98^{\circ} \mathrm{C} / 10 \mathrm{sec}$ and $58^{\circ} \mathrm{C} / 20 \mathrm{sec}$. After an initial step of $95^{\circ} \mathrm{C} / 10 \mathrm{sec}$ and $65^{\circ} \mathrm{C} / 10 \mathrm{sec}$, melting curves were generated from $65^{\circ} \mathrm{C}$ to $95^{\circ} \mathrm{C}$ in increments of $0.3^{\circ} \mathrm{C} /$ cycle. Melting profiles were analyzed with Precision Melt Analysis ${ }^{\mathrm{TM}}$ Software (Bio-Rad Laboratories Inc., Hercules, CA, USA).

\section{Obsessive behavior assessment}

Obsessive behaviors were measured by a single administration of a 30-item Korean translation of the Maudsley Obsessive-Compulsive Inventory (MOCI), one of the psychological tests most commonly used for assessing obsessive and compulsive symptoms [26]. MOCI is a self-reporting questionnaire with dichotomized scores ("present" or "not present"), yielding a maximum MOCI total score of thirty. Based on thirty items, the following four clinical subscales have been constructed: checking $(9$ items), cleaning (11 items), delay (7 items), and suspicion (7 items). Four of the items contribute to the score on two of the subscales. The Korean version has shown to have good internal consistency, good testretest reliability, good discrimination, and convergent validity [27]. All participants completed the Korean translation of the MOCI and were classified into two groups based on their total MOCI score. We determined the cutoff of the MOCI total score for the obsessive group as 17, based on two standard deviations from the mean total score in healthy controls recruited from our study because the original study did not suggest it. Among a total of 292 participants, 274 were classified into the non-obsessive group and 18 into the obsessive group.

\section{Statistical analysis}

Statistical analyses were carried out with IBM SPSS 22.0 for Windows (IBM Corporation, Armonk, NY, USA) and SNPAlyze (version 7, DYNACOM, Chiba, Japan). The presence of HardyWeinberg equilibrium, linkage disequilibrium, and a case-control study using dominant, recessive, and genotypic hereditary models were conducted using SNPAlyze software. Comparison of MOCI scores between the 3 genotype groups was performed using analysis of variance (ANOVA), followed by pairwise comparison using Scheffe's method. In case of nonparametric groups, the Kruskal-Wallis test was used instead. The results of analysis were considered at nominal significance $(\mathrm{p}<0.05)$.

\section{RESULTS}

\section{Clinical characteristics of participants}

Among the total 292 participants, 187 were male and 105 were female. The mean (mean \pm standard deviation) age and total MOCI score were $22.92 \pm 2.26$ and $7.86 \pm 5.03$, respectively. The non-obsessive group consisted of 178 men and 96 women. The mean clinical characteristics of the non-obsessive group were as follows: age $22.95 \pm 2.29$, body mass index (BMI) $21.42 \pm 3.41$, and years of education $15.6 \pm 1.47$, respectively. Among the 18 participants in the obsessive group, 9 were male and 9 were female. Their mean clini- 
cal characteristics were as follows: age $22.50 \pm 1.76$, BMI $21.73 \pm$ 2.90 , and years of education $15.2 \pm 1.51$. No significant difference was found in age, BMI, or years of education between the two groups. The scores of total MOCI and its subscales (checking, cleaning, suspicion, and delay) in the non-obsessive group are shown in Table 1 . The total and subscale scores were significantly higher in the obsessive group than in the non-obsessive group $(\mathrm{p}<0.001)$.

The VNTR and all SNPs analyzed in this study were at HardyWeinberg equilibrium and had minor allele frequencies over $5 \%$. In the present study, six circadian gene polymorphisms (PER3 rs57875989, NPAS2 rs2305160, ARNTL rs2278749, CLOCK rs1802160, PER2 rs2304672, PER2 rs934945) were analyzed in 292 participants. The distribution of allele and genotype frequen-

Table 1. Clinical characteristics of the study participants

\begin{tabular}{lccc}
\multicolumn{1}{c}{ Characteristics } & $\begin{array}{c}\text { Non-obsessive } \\
\text { group }\end{array}$ & $\begin{array}{c}\text { Obsessive } \\
\text { group }\end{array}$ & p-value \\
Female* $^{*}$ & $96(35)$ & $9(50)$ & 0.213 \\
Male* & $178(65)$ & $9(50)$ & \\
Age $(\mathrm{year})^{\dagger}$ & $22.95 \pm 2.29$ & $22.50 \pm 1.76$ & 0.420 \\
$\mathrm{BMI}\left(\mathrm{kg} / \mathrm{m}^{2}\right)^{\dagger}$ & $21.42 \pm 3.41$ & $21.73 \pm 2.90$ & 0.720 \\
Educational years $^{\dagger}$ & $15.6 \pm 1.47$ & $15.2 \pm 1.51$ & 0.274 \\
Total MOCI score $^{\dagger}$ & $7.08 \pm 4.10$ & $19.7 \pm 2.37$ & $<0.001$ \\
Checking $^{\dagger}$ & $1.85 \pm 1.46$ & $5.11 \pm 0.832$ & $<0.001$ \\
Cleaning $^{\dagger}$ & $1.39 \pm 1.39$ & $5.56 \pm 1.76$ & $<0.001$ \\
Suspicion $^{\dagger}$ & $2.78 \pm 1.82$ & $5.56 \pm 0.92$ & $<0.001$ \\
Delay $^{\dagger}$ & $1.06 \pm 1.05$ & $3.50 \pm 1.10$ & $<0.001$ \\
\hline
\end{tabular}

Data are expressed on mean \pm SD or $\mathrm{n}(\%) .{ }^{*}$ results of chi-square test, ${ }^{\dagger}$ results of independent t-tests. MOCI: Maudsley Obsessive-Compulsive Inventory, BMI: body mass index cies of the VNTR and SNPs in the obsessive and non-obsessive groups is presented in Table 2.

Association analysis of circadian gene variations on obsessive compulsive behaviors

The genotype frequencies of PER3 rs57875989 in the obsessive and non-obsessive groups are shown in Table 2. The association analysis results indicate a significant difference in PER3 rs57875989 genotype frequencies between the two groups under a recessive hereditary model [Akaike's information criterion $(\mathrm{AIC})=2.1818$, $\mathrm{p}=0.005175$ ] and a genotypic hereditary model $(\mathrm{AIC}=0.2473, \mathrm{p}=$ $0.0195)$. However, after multiple comparison correction, only the recessive model was significant [False Discovery Rate (FDR) q-value $=0.0327$ ] (Table 2).

The genotypic frequencies of ARNTL rs2278749 in the obsessive and non-obsessive groups are also shown in Table 2. The association analysis results indicate a significant difference of $A R N$ $T L$ rs2278749 genotype frequencies under a recessive hereditary model (AIC=5.7245, p=0.0196), but it is not significant after multiple comparison correction (FDR q-value=0.1239). For the other circadian gene SNPs (NPAS2 rs2305160, CLOCK rs1801260, PER2 rs2304672, PER2 rs934945), the frequencies of these SNPs indicate no significant difference between the obsessive and nonobsessive groups based on all three hereditary models $(\mathrm{p}<0.05)$.

For PER3 rs57875989 and ARNTL rs2278749, we analyzed the differences in MOCI total score and its subscales between the genotypes. The mean scores of total MOCI and its subscales (checking, cleaning, suspicion, and delay) in PER3 rs57875989 genotypes are shown in Table 3. No significant differences in MOCI total score and its subscales between the genotypes of PER3 rs57875989 were

Table 2. Genotype distribution and association analysis of circadian gene variations on obsessive compulsive behaviors

\begin{tabular}{|c|c|c|c|c|c|c|}
\hline & & 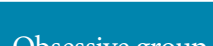 & 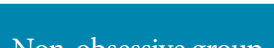 & Dominant model & Recessive model & Genotype model \\
\hline & & Uosessive group & Non-obsessive group & p-value (FDR q-value) & p-value (FDR q-value) & $\overline{p \text {-value (FDR q-value) }}$ \\
\hline PER3 & $4 / 4$ & $12(66.7)$ & $210(76.6)$ & 0.3369 & $0.005175^{*}$ & $0.0195^{*}$ \\
\hline rs57875989 & $4 / 5$ & $4(22.2)$ & $60(21.9)$ & $(0.7092)$ & $(0.0327)^{*}$ & $(0.1229)$ \\
\hline & $5 / 5$ & $2(11.1)$ & $4(1.5)$ & & & \\
\hline NPAS2 & GG & $13(72.2)$ & $181(66.1)$ & 0.3241 & 0.5507 & 0.4164 \\
\hline rs2305160 & GA & $4(22.2)$ & $88(32.1)$ & $(0.7092)$ & $(0.7839)$ & $(0.7965)$ \\
\hline & AA & $1(5.6)$ & $5(1.8)$ & & & \\
\hline ARNTL & $\mathrm{CC}$ & $17(94.4)$ & $186(67.9)$ & $0.0196^{*}$ & 0.6984 & 0.0812 \\
\hline rs2278749 & CT & $1(5.6)$ & $78(28.5)$ & $(0.1239)$ & $(0.7839)$ & $(0.2566)$ \\
\hline & TT & $0(0)$ & $10(3.6)$ & & & \\
\hline CLOCK & TT & $14(77.8)$ & $229(83.6)$ & 0.7374 & 0.8688 & 0.8922 \\
\hline rs 1801260 & TC & $4(22.2)$ & $41(15)$ & $(0.7762)$ & $(0.7839)$ & $(0.805)$ \\
\hline & $\mathrm{CC}$ & $0(0)$ & $4(1.5)$ & & & \\
\hline PER2 & GG & $14(77.8)$ & $230(83.9)$ & 0.4699 & 0.6548 & 0.6306 \\
\hline rs2304672 & GC & $4(22.2)$ & 40 (14.6) & $(0.742)$ & $(0.7839)$ & $(0.7965)$ \\
\hline & $\mathrm{CC}$ & $0(0)$ & $3(1.1)$ & & & \\
\hline PER2 & GG & $8(44.4)$ & $118(43.1)$ & 0.9193 & 0.3465 & 0.6259 \\
\hline rs934945 & GA & $9(50)$ & $119(43.4)$ & $(0.8295)$ & $(0.7839)$ & $(0.7965)$ \\
\hline & AA & $1(5.6)$ & $36(13.1)$ & & & \\
\hline
\end{tabular}

Data are expressed on n (\%). Case-control study was conducted by SNPAlyze (ver 7, DYNACOM). *p-value<0.05. FDR: False Discovery Rate 
Table 3. Comparison of $\mathrm{MOCl}$ total score and its subscales between genotypes of PER3 rs57875989 and ARNTL rs2278749

\begin{tabular}{|c|c|c|c|c|c|c|c|}
\hline & \multirow{2}{*}{ Genotype } & \multirow{2}{*}{$\mathrm{N}(\%)$} & \multicolumn{5}{|c|}{ MOCI score } \\
\hline & & & Checking & Cleaning & Suspicion & Delay & Total \\
\hline \multirow[t]{4}{*}{ PER3 rs57875989' } & $4 / 4$ & $222(76)$ & $2.03 \pm 1.61$ & $1.65 \pm 1.76$ & $2.93 \pm 1.89$ & $1.21 \pm 1.18$ & $7.82 \pm 4.94$ \\
\hline & $4 / 5$ & $64(21.9)$ & $2.06 \pm 1.70$ & $1.47 \pm 1.46$ & $3.03 \pm 1.93$ & $1.22 \pm 1.32$ & $7.77 \pm 5.21$ \\
\hline & $5 / 5$ & $6(2.1)$ & $2.83 \pm 1.72$ & $3.33 \pm 2.73$ & $3.17 \pm 2.14$ & $1.17 \pm 1.33$ & $10.5 \pm 6.95$ \\
\hline & p-value & & 0.454 & 0.156 & 0.838 & 0.966 & 0.569 \\
\hline \multirow[t]{4}{*}{$A R N T L$ rs2278749 } & CC & CC (203) & $2.22 \pm 1.67$ & $1.78 \pm 1.80$ & $3.03 \pm 1.93$ & $1.28 \pm 1.25$ & $8.32 \pm 5.34$ \\
\hline & $\mathrm{CT}$ & CT (79) & $1.66 \pm 1.52$ & $1.39 \pm 1.53$ & $2.76 \pm 1.83$ & $1.03 \pm 1.6$ & $6.82 \pm 4.18$ \\
\hline & TT & TT (10) & $1.70 \pm 1.25$ & $0.80 \pm 1.55$ & $3.00 \pm 1.63$ & $1.30 \pm 1.25$ & $6.80 \pm 3.52$ \\
\hline & $\mathrm{p}$-value & & $0.026^{*}$ & $0.038^{*}$ & 0.561 & 0.272 & 0.052 \\
\hline Post-hoc comparison & & & $\mathrm{CC}>\mathrm{CT}^{*}$ & & & & \\
\hline
\end{tabular}

Data are expressed on mean \pm SD or $\mathrm{n}(\%)$. Post-hoc comparison was done by Scheffe test. ${ }^{*}$ p-value $<0.05$, ${ }^{\dagger}$ results of Kruskal-Wallis test, ${ }^{\ddagger}$ results of analysis of variance. MOCI: Maudsley Obsessive-Compulsive Inventory

found.

In the case of ARNTL rs2278749, the mean scores of total MOCI and its subscales (checking, cleaning, suspicion, and delay) are also shown in Table 3. ANOVA shows significant differences in checking and cleaning subscales $(\mathrm{p}<0.05)$ between the genotypes of ARNTL rs2278749. In subgroup analysis, the checking subscale scored significantly higher in the CC genotype than the CT genotype.

\section{DISCUSSION}

Emerging evidence indicates that circadian rhythm has an important role in OCD, but the relationship between circadian rhythm and OCD is still unclear $[28,29]$. Previous studies on the role of circadian rhythms in obsessive-compulsive disorder have been focused on chronotype [17], delayed sleep phase disorder $[15,30]$, and hormonal changes [5-7,31].

The main finding of this study is that circadian gene variants are associated with obsessive-compulsive behaviors. Specifically, PER3 rs57875989 VNTR and ARNTL rs2278749 SNP showed significant differences in genotype frequencies between the obsessive and non-obsessive groups. There were significant differences in the checking and cleaning subscale scores between genotypes of ARNTL rs2278749 SNP.

In the previous study, PER3 rs57875989 5/5 genotype had worse performance in measures of planning performance and working memory, and lower reaction times in psychomotor vigilance tasks [32-34]. In our results, the 5/5 genotype had the highest total MOCI score of 10.5 , while the $4 / 4$ and $4 / 5$ genotypes scored 7.82 and 7.77, respectively. As planning is an important component of obsessive symptoms [35], these results are consistent with those of previous studies.

In this study, the ARNTL rs2278749 CC genotype was associated with more severe obsessive behaviors. As a previous study showed that ARNTL gene polymorphism plays a role in eveningness [21], this may explain the association between eveningness and obsessive symptoms $[14,18,30]$.

Moreover, circadian rhythm consists of a complicated molecu- lar network of a number of circadian genes, rather than on individual circadian genes, and interactions between circadian genes may be more important for the occurrence and development of obsessive-compulsive symptoms. Further studies are needed to find the link between circadian rhythm and obsessive-compulsive symptoms.

Serotonin is an important regulator of the mammalian circadian clock of the suprachiasmatic nucleus. Serotonin in raphe nuclei and intergeniculate leaflets triggers phase shifts [36,37]. Circadian rhythm and serotonin are very closely related to each other. Although the mechanism by which serotonin causes OCD is not known, it is well-known that low serotonin and OCD are related. Evidence shows that the most effective treatment for OCD is to increase serotonin. Antidepressants, called selective serotonin reuptake inhibitors, are the most commonly prescribed drugs for OCD [38].

Based on previous studies and the association between circadian gene variants and obsessive-compulsive behaviors in a normal population reported here, we hypothesized that the effect of circadian gene variants on serotonin regulation may affect the obsessive-compulsive behavior in a normal population.

Further studies are warranted to evaluate the possible involvement of as-yet-undiscovered genes in obsessive-compulsive behaviors. We cannot exclude population stratification bias; however, the Korean population is characterized by a relatively high degree of genetic homogeneity [39]. Therefore, population stratification bias is unlikely in our sample. Furthermore, our sample was composed of young individuals of the same ethnic group, which itself is a very homogenous group. Therefore, many confounding factors were excluded in this study.

As this study is conducted on a young healthy population, and not on those with OCD, the results on this study should be interpreted carefully. Nevertheless, it is meaningful that even in a healthy population, the association between circadian gene variations and obsessive-compulsive behavior could be seen. Further study on OCD patients and healthy controls may be needed. However, this study is worthwhile in the sense that obsessive-compulsive behaviors are not directly related to the mental illness of OCD. 
In conclusion, this study is the first to reveal an association between circadian gene (ARNTL and PER3) variants and obsessivecompulsive behaviors in a Korean population. Further studies are needed to explain the link between the circadian genes and obsessive-compulsive behavior.

\section{Acknowledgments}

This study was supported by the Korea Health 21 R\&D Project, funded by the National Research Foundation of Korea (2017M3 A9F1031220).

\section{Conflicts of Interest}

The authors have no potential conflicts of interest to disclose.

\section{Author Contributions}

Conceptualization: Heon-Jeong Lee. Formal analysis: Heon-Jeong Lee, Chul-Hyun Cho, Yujin Lee, Jaegwon Jeong. Funding acquisition: Heon-Jeong Lee. Investigation: Heon-Jeong Lee, Chul-Hyun Cho, Yujin Lee, Jaegwon Jeong. Methodology: Heon-Jeong Lee, Jaegwon Jeong. Project administration: Heon-Jeong Lee. Resources: Heon-Jeong Lee. Supervision: Heon-Jeong Lee, ChulHyun Cho, Yujin Lee. Software: Heon-Jeong Lee. Validation: Heon-Jeong Lee, Chul-Hyun Cho, Yujin Lee, Jaegwon Jeong. Visualization: Jaegwon Jeong. Writing-original draft: Jaegwon Jeong. Writing — review and editing: Heon-Jeong Lee, Chul-Hyun Cho, Yujin Lee, Jaegwon Jeong.

\section{ORCID iDs}

Heon-Jeong Lee (D)

https://orcid.org/0000-0002-9560-2383

Jaegwon Jeong (iD

https://orcid.org/0000-0003-1152-833X

\section{REFERENCES}

1. Lee HJ, Son GH, Geum D. Circadian rhythm hypotheses of mixed features, antidepressant treatment resistance, and manic switching in bipolar disorder. Psychiatry Investig 2013;10:225-232.

2. Takaesu Y. Circadian rhythm in bipolar disorder: a review of the literature. Psychiatry Clin Neurosci 2018;72:673-682.

3. Chouinard S, Poulin J, Stip E, Godbout R. Sleep in untreated patients with schizophrenia: a meta-analysis. Schizophr Bull 2004;30:957-967.

4. Glickman G. Circadian rhythms and sleep in children with autism. Neurosci Biobehav Rev 2010;34:755-768.

5. Catapano F, Monteleone P, Fuschino A, Maj M, Kemali D. Melatonin and cortisol secretion in patients with primary obsessive-compulsive disorder. Psychiatry Res 1992;44:217-225.

6. Kluge M, Schüssler P, Künzel HE, Dresler M, Yassouridis A, Steiger A. Increased nocturnal secretion of ACTH and cortisol in obsessive compulsive disorder. J Psychiatr Res 2007;41:928-933.

7. Millet B, Touitou Y, Poirier MF, Bourdel MC, Hantouche E, Bogdan A, et al. Plasma melatonin and cortisol in patients with obsessive-compulsive disorder: relationship with axillary temperature, physical activity, and clinical symptoms. Biol Psychiatry 1998;44:874-881.

8. Arriaga F, Lara E, Matos-Pires A, Cavaglia F, Bastos L. Diagnostic relevance of sleep complaints in anxiety and mood disorders. Eur Psychiatry 1995; 10:386-390.

9. Insel TR, Gillin JC, Moore A, Mendelson WB, Loewenstein RJ, Murphy DL. The sleep of patients with obsessive-compulsive disorder. Arch Gen Psychia- try 1982;39:1372-1377.

10. Robinson D, Walsleben J, Pollack S, Lerner G. Nocturnal polysomnography in obsessive-compulsive disorder. Psychiatry Res 1998;80:257-263.

11. Kluge M, Schüssler P, Dresler M, Yassouridis A, Steiger A. Sleep onset REM periods in obsessive compulsive disorder. Psychiatry Res 2007;152:29-35.

12. Díaz-Román A, Perestelo-Pérez L, Buela-Casal G. Sleep in obsessive-compulsive disorder: a systematic review and meta-analysis. Sleep Med 2015; 16:1049-1055.

13. Coles ME, Schubert JR, Sharkey KM. Delayed bedtimes and obsessive-compulsive symptoms. Behav Sleep Med 2012;10:258-265.

14. Turner J, Drummond LM, Mukhopadhyay S, Ghodse H, White S, Pillay A, et al. A prospective study of delayed sleep phase syndrome in patients with severe resistant obsessive-compulsive disorder. World Psychiatry 2007;6: 108-111.

15. Schubert JR, Coles ME. Obsessive-compulsive symptoms and characteristics in individuals with delayed sleep phase disorder. J Nerv Ment Dis 2013; 201:877-884.

16. Tan O, Metin B, Ünsalver BÖ, Sayar GH. Seasonal mood changes in patients with obsessive-compulsive disorder. Psychiatry Res 2017;258:166-170.

17. Cox RC, Tuck B, Olatunji BO. The role of eveningness in obsessive-compulsive symptoms: cross-sectional and prospective approaches. J Affect Disord 2018;235:448-455.

18. Coles ME, Wirshba CJ, Nota J, Schubert J, Grunthal BA. Obsessive compulsive disorder prevalence increases with latitude. J Obsessive Compuls Relat Disord 2018;18:25-30

19. Takahashi JS, Hong HK, Ko CH, McDearmon EL. The genetics of mammalian circadian order and disorder: implications for physiology and disease. Nat Rev Genet 2008;9:764-775.

20. Lee HJ, Kim L, Kang SG, Yoon HK, Choi JE, Park YM, et al. PER2 variation is associated with diurnal preference in a Korean young population. Behav Genet 2011;41:273-277.

21. Song HM, Cho CH, Lee HJ, Moon JH, Kang SG, Yoon HK, et al. Association of CLOCK, ARNTL, PER2, and GNB3 polymorphisms with diurnal preference in a Korean population. Chronobiol Int 2016;33:1455-1463.

22. Lee HJ, Paik JW, Kang SG, Lim SW, Kim L. Allelic variants interaction of CLOCK gene and G-protein beta3 subunit gene with diurnal preference. Chronobiol Int 2007;24:589-597.

23. Ojeda DA, Perea CS, Niño CL, Gutiérrez RM, López-León S, Arboleda H, et al. A novel association of two non-synonymous polymorphisms in PER2 and PER3 genes with specific diurnal preference subscales. Neurosci Lett 2013;553:52-56

24. Kim HI, Lee HJ, Cho CH, Kang SG, Yoon HK, Park YM, et al. Association of CLOCK, ARNTL, and NPAS2 gene polymorphisms and seasonal variations in mood and behavior. Chronobiol Int 2015;32:785-791.

25. Alvaro PK, Roberts RM, Harris JK, Bruni O. The direction of the relationship between symptoms of insomnia and psychiatric disorders in adolescents. J Affect Disord 2017;207:167-174.

26. Hodgson RJ, Rachman S. Obsessional-compulsive complaints. Behav Res Ther 1977;15:389-395.

27. Min BB, Won HT. Reliability and validity of the Korean translations of Maudsley obsessional-compulsive inventory and Padua inventory. Korean J Clin Psychol 1999;18:163-182.

28. Cox RC, Olatunji BO. Circadian rhythms in obsessive-compulsive disorder: recent findings and recommendations for future research. Curr Psychiatry Rep 2019;21:54.

29. Lange KW, Lange KM, Hauser J, Tucha L, Tucha O. Circadian rhythms in obsessive-compulsive disorder. J Neural Transm (Vienna) 2012;119:10771083.

30. Nota JA, Sharkey KM, Coles ME. Sleep, arousal, and circadian rhythms in adults with obsessive-compulsive disorder: a meta-analysis. Neurosci Biobehav Rev 2015;51:100-107.

31. Monteleone P, Catapano F, Tortorella A, Di Martino S, Maj M. Plasma melatonin and cortisol circadian patterns in patients with obsessive-compulsive disorder before and after fluoxetine treatment. Psychoneuroendocrinology 1995;20:763-770.

32. González-Giraldo Y, González-Reyes RE, Mueller ST, Piper BJ, Adan A, Forero DA. Differences in planning performance, a neurocognitive endophenotype, are associated with a functional variant in PER3 gene. Chronobiol Int 2015;32:591-595. 
33. Groeger JA, Viola AU, Lo JC, von Schantz M, Archer SN, Dijk DJ. Early morning executive functioning during sleep deprivation is compromised by a PERIOD3 polymorphism. Sleep 2008;31:1159-1167.

34. Lo JC, Groeger JA, Santhi N, Arbon EL, Lazar AS, Hasan S, et al. Effects of partial and acute total sleep deprivation on performance across cognitive domains, individuals and circadian phase. PLoS One 2012;7:e45987.

35. Sullivan JR, Riccio CA, Castillo CL. Concurrent validity of the tower tasks as measures of executive function in adults: a meta-analysis. Appl Neuropsychol 2009;16:62-75.

36. Page TL. Serotonin phase-shifts the circadian rhythm of locomotor activity in the cockroach. J Biol Rhythms 1987;2:23-34.

37. Smith VM, Jeffers RT, Antle MC. Serotonergic enhancement of circadian responses to light: role of the raphe and intergeniculate leaflet. Eur J Neurosci 2015;42:2805-2817.

38. Pittenger C, Bloch MH. Pharmacological treatment of obsessive-compulsive disorder. Psychiatr Clin North Am 2014;37:375-391.

39. Han GR, Lee YW, Lee HL, Kim SM, Ku TW, Kang IH, et al. A Korean population study of the nine STR loci FGA, VWA, D3S1358, D18S51, D21S11, D8S1179, D7S820, D13S317 and D5S818. Int J Legal Med 2000;114:41-44. 